\title{
MEASUREMENT OF IODINE INTAKE AND ITS RELATIONSHIP WITH SOME PERSONAL CHARACTERISTICS OF SOME LEARNING DISABILITY FEMALE STUDENTS (7-14 YEARS) IN THE CENTRAL REGION OF SAUDI ARABIA
}

(Received: 15.10.2006)

\author{
By \\ A. A. Al-Shoshan and A. A. Al-Kahtani \\ Food Science and Nutrition Department, College of Food Sciences and Agriculture, King Saud \\ University, Riyadh, Saudi Arabia
}

\begin{abstract}
This study was conducted to assess iodine intake by some learning disability female students (7-14 years) in the Central Region of Saudi Arabia and its relationship with their personal characteristics. Data were collected through personal interview using a questionnaire. As simple random sample of 340 learning disability female students was selected. Iodine consumption and iodine secretion rate was performed by analyzing urine samples taken from the students. Percentages, mathematical mean, t-test, analysis of variance and L.S.D. analysis were used to analyze the data statistically.

The results showed that $25.4 \%$ have low rate of iodine secretion in urine. In addition, the results revealed that iodine consumption increased with the increasing level of education of $60^{\text {th }}$ father's and mother's students and also increased with the moderate level of family income. Analysis of variance showed that there was a significant relationship between the groups in urine secretion rate and between the groups of iodine consumption according to mother's and father's education and family income.

The research recommended that female students should depend more on food rich in iodine such as fish and other foods rich in iodine.
\end{abstract}

Key words: iodine intake, learning disability, Saudi Arabia.

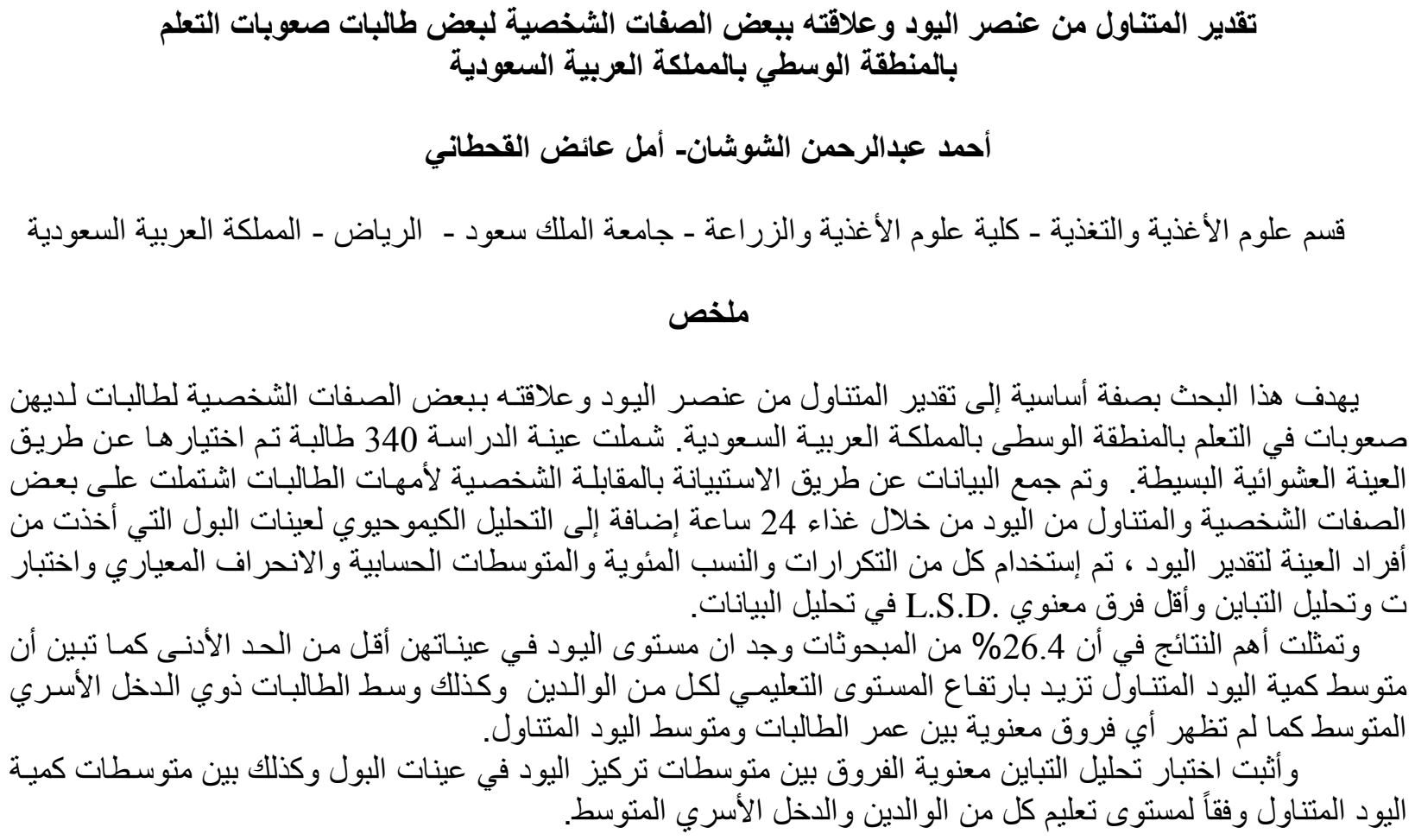

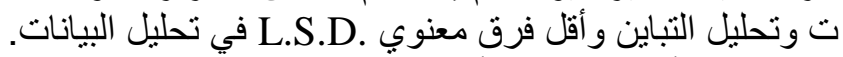

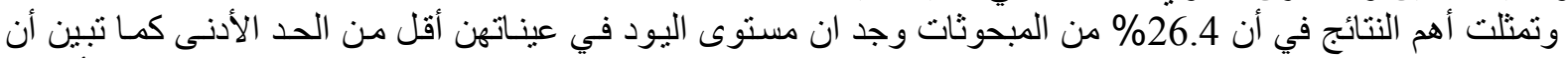

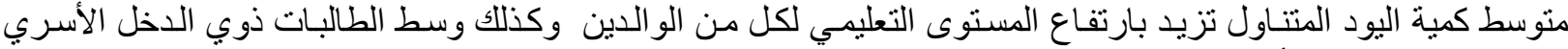

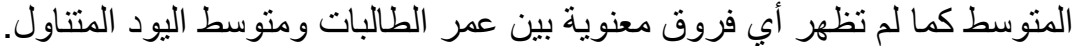

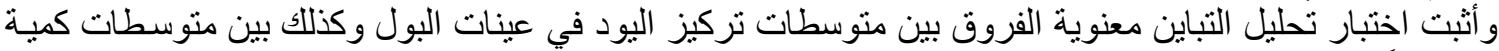

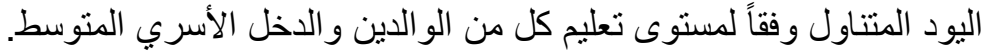


و أوصت الدراسـة ضرورة تنـاول الطالبـات المجموعات الغذائية الغنية بعنصر اليود مثل الأسماك وبعض الأغذية

الأخري.

يوجد اليود في الغذاء بصـورة أساسية في يـود غير مير

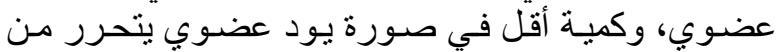

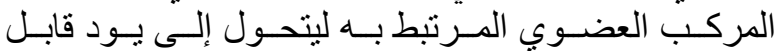

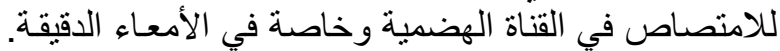

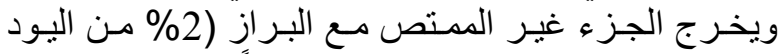

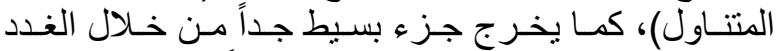

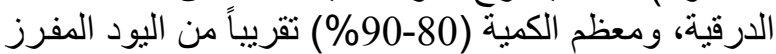

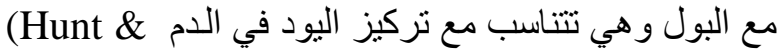
.Groof, 1990)

يؤدي نقص اليود إلى حدوث اضطر ابات ذات تأثثرات

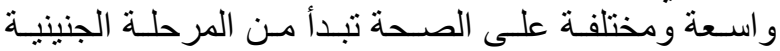

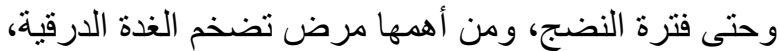

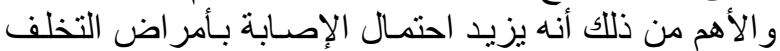

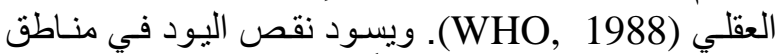

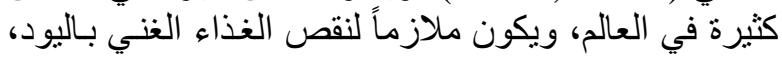

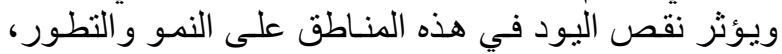

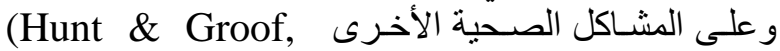
.1990)

بالمقابـل فقــ تتضــــم الغـدة الدرقيـة مـن جـر اء زيــادة

المتنـاول، وخصوصـاً الجرعـات الدو ائية

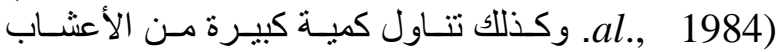

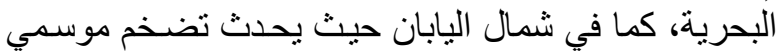

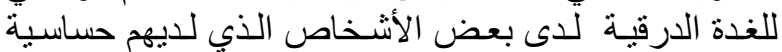

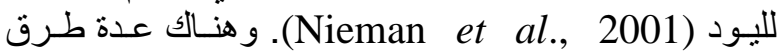

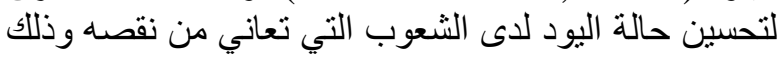

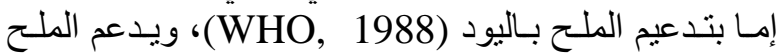
بإضـافة يوديد البوتانسيوم Potassium iodide) KI) أو الو (Potan أيودات البوتاسيوم (Potassium iodate) $\mathrm{KIO}_{3}$

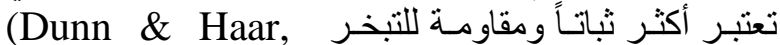

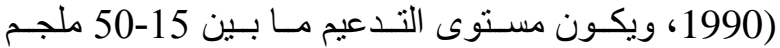

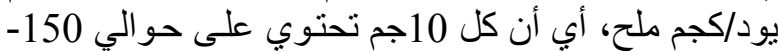
500 ميكروجرام يود (WHO, 1988). أما الطريقة الثانية

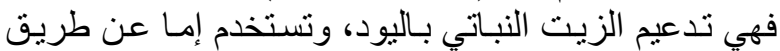

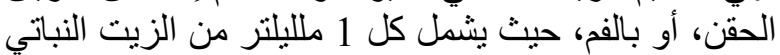

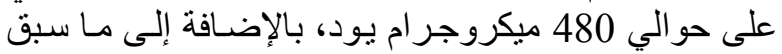

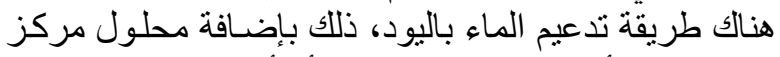

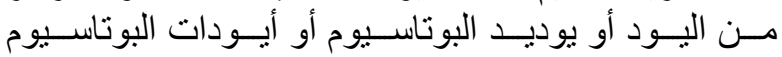

.(WHO, 1988; Dunn \& Haar, 1990) يفضـلـ في كثيـر مـن الـدول المتقدمـة تـدعيم الخبـز

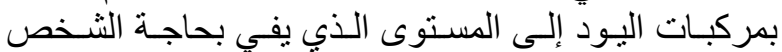
اليومية (Brasward et al., 1995). تثشير كثير من الدراسات إلى أن قباس اليود المفرز في

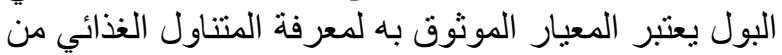

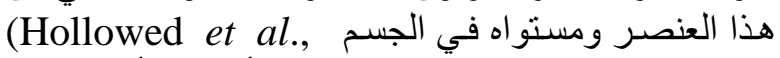
(1998; Delange et al., 2002) انخفاض تركيزه في البــول يعني انخفاض

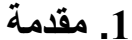

يعتبر اليود من العناصر الغذائية المهمـة لجسم الإنسـان

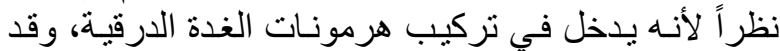

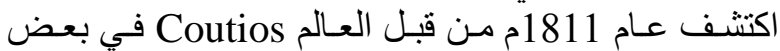

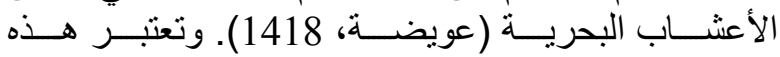

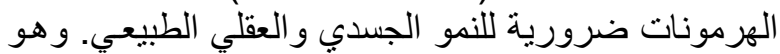

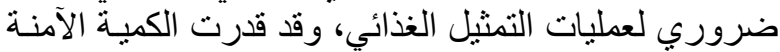

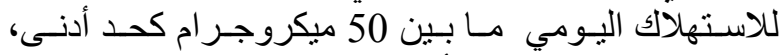

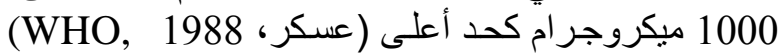
1991; Hunt \& Groof, 1990;

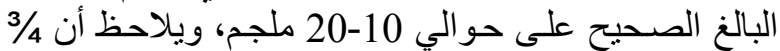
هذه الكمية مركزة في الغدة الدرقية، ويكون تركيز العنصر

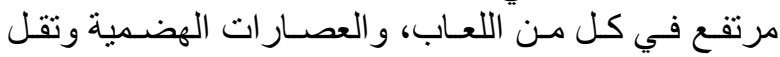

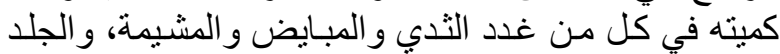

.(Nieman et al., 2001)

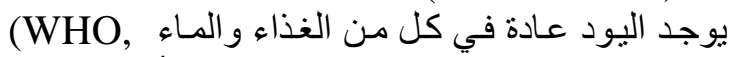

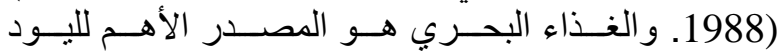

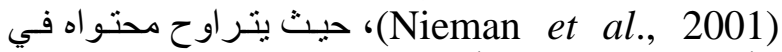

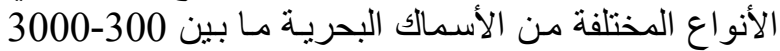

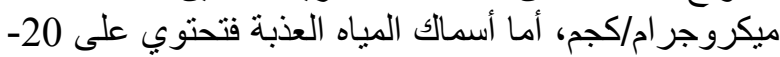

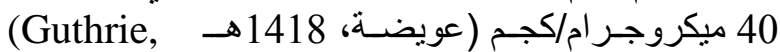
.1983; Hunt \& Groof, 1990 ; وتعتبر الألبان مصدر اً جيداً لليود حيث يتر اوح محتو اهـا

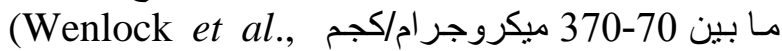

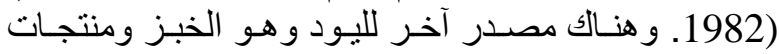

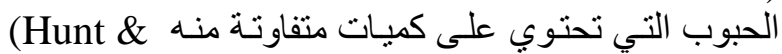
Groof, 1990)

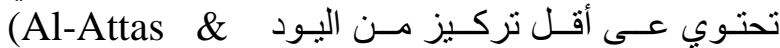
.Sulimani, 1993)

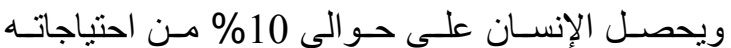

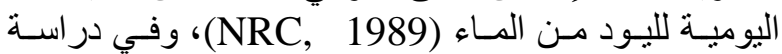

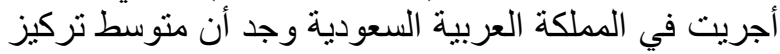

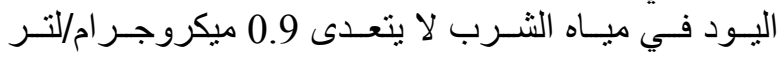

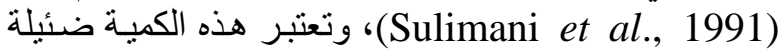

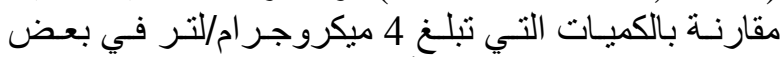

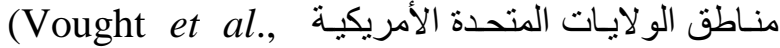
.1967)

حددت هيئة الغذاء و التغذية في مجلس الأبحاث الوطني الأمريكي FNB and NRC (1989) المقررات الغذاتئية الغئية

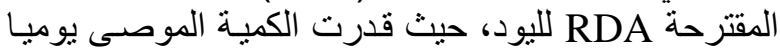

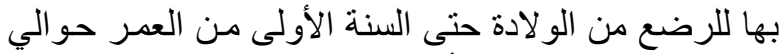

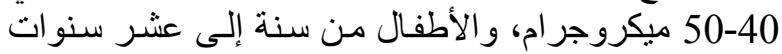

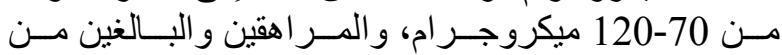

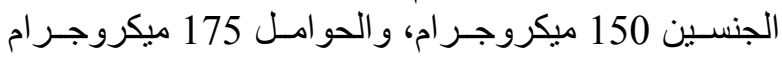

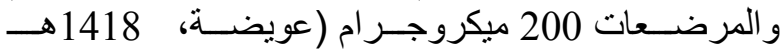
.(NRC, 1989; 
اعتمد على الاستبيان بالمقابلة الشخصية لجمع البيانـات

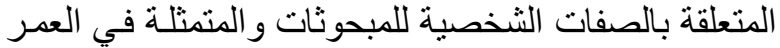

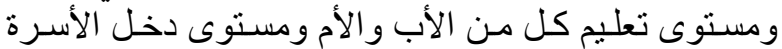

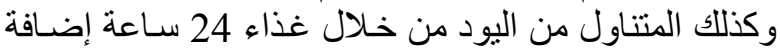

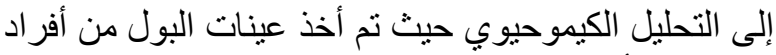

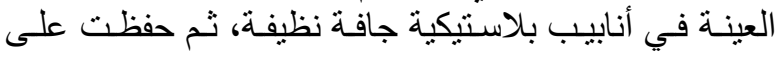
درجة 20×م لحين إجر اء التحليل اللازم و المتمثل في تقدير

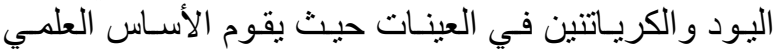

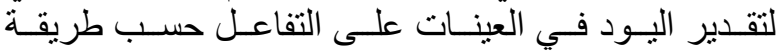
بو، Sandell \& Klothoff, 1937

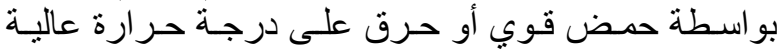

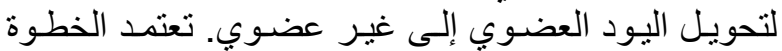

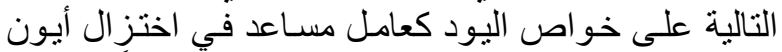

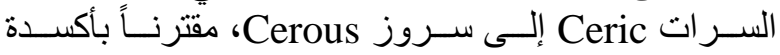

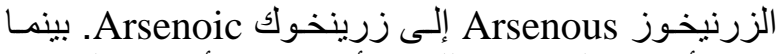

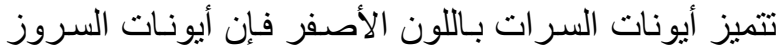

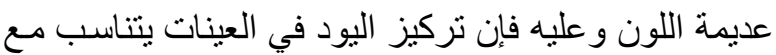

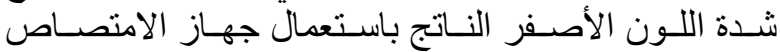
الضـوئي Spectrophotometer على موجـة طولهـا لونها 405 (Hunt \& Groof, 1990; Dunn et al., نسانومتر

وتـم تقدير الكريـاتنين في البـول عـن طريـق اسـتخدام

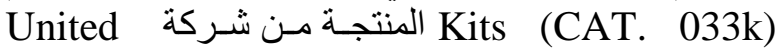
Diagnostics Industry (UDI)

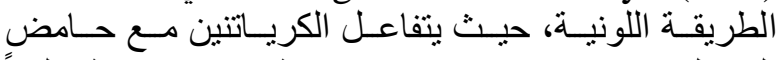
البكريك Picric acid في وجود وسط قاعدي، ويعطي لوناً

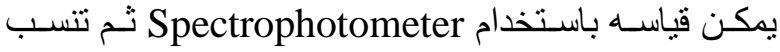

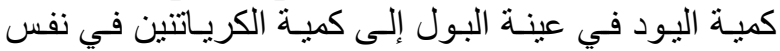

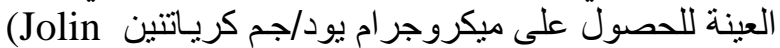
\& Escobar del Ray, 1956) 3.3. التحليل الإحصائي

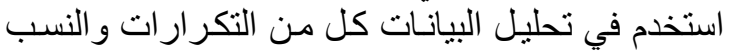
المئوية و الانحر اف المعيـاري و اختبار (ت) وتحليـل التباين .L.S.D. و

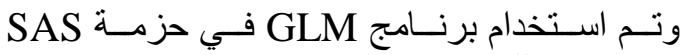

للتحليل الإحصائي للبيانات (SAS, 1995).

\section{4. نتائج البحث ومناقشتها} 1.4. تركيز اليود في العينات

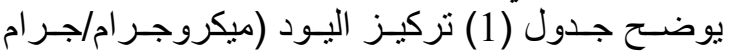
كرياتنين) في عينات البول لطالبات صعوبات التعلم وتظهر النتائج أن مستوى اليود 26.4\% منهن في عيناتهن أقل من الته

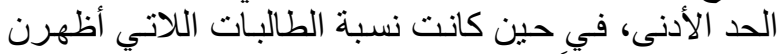

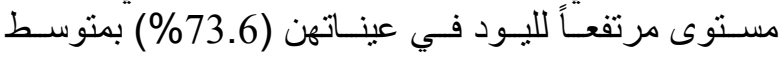
تر اوح ما بين 215.29-40.7 ميكروجر ام/جر ام كرياتنين.

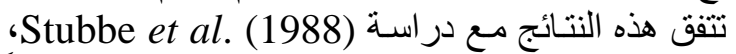

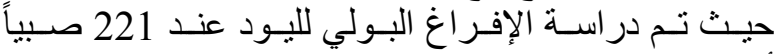
أعمار هم بين 6-12 سنة في المملكة العربية السعودية لإنودية
كميـة اليود المتاح، وارتفـاع مستو اه في البول يـدل على متناول وافر من اليود (Guthrie, 1983).

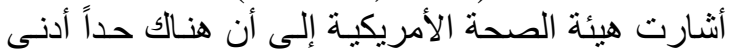

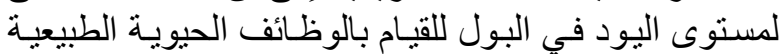

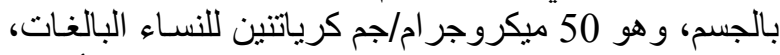

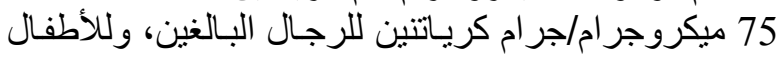

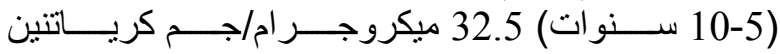

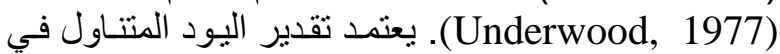

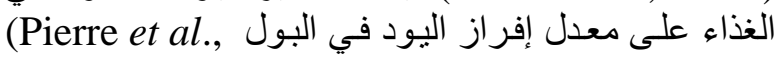

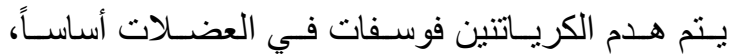

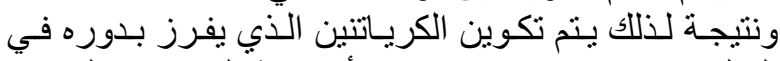

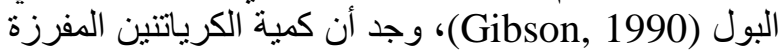

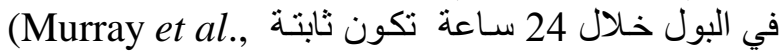
(1991) ولقد شاع استخدام نسبة وجود اليود إلى الكرياتنين منذ أكثر من عشرين عامـاً (Iodine/creatinine ratio) (Underwood, 1977; في كثيـر مسن الأبحـاث، .Gushurst et al., 1984; Strove \& Ohlen, 1990) و انطلاقاً من أن صعوبات التعلم أصبحت قضبية تستحوذ

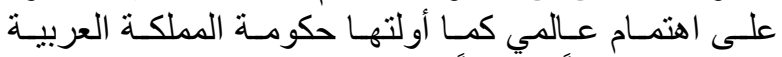

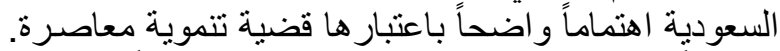

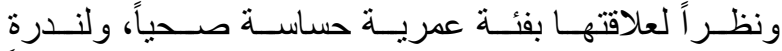
الدر اسات التي أجربت داخل المملكة و العالم العربي عمومـاً

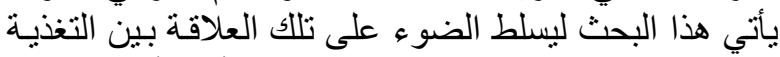

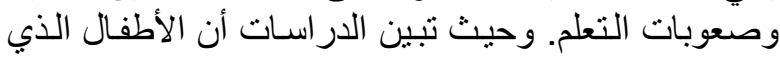

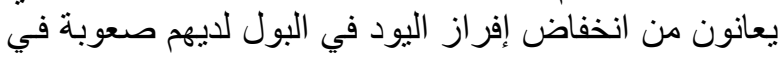
القر اءة و الهجاء و الرياضيات (Sayed et al., 1999). وأن المملكة قد صنفت من ضمن مناطق النقص الطفيف في

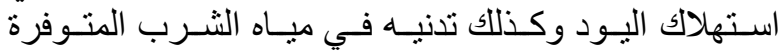

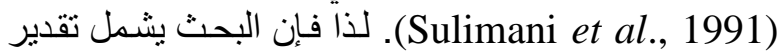
المتناول من عنصر اليود لعينة من طالبات صـعوبات التعلم

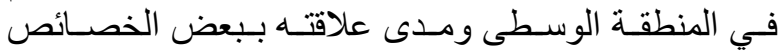
الثخصية للمبحوثات.

\section{2. - 2. أهداف البحث}

يهذف هذا البحث إلى الآتي:

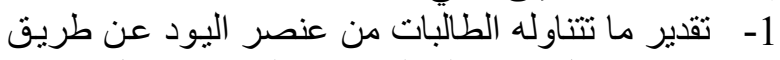

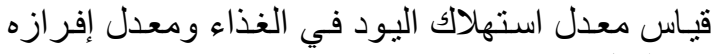

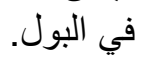

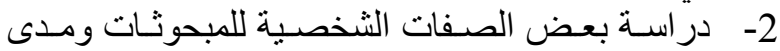

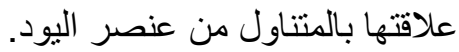

\section{3. المواد وطرق العمل Materials and Methods} 1.3 عينة البحث

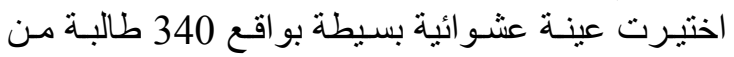

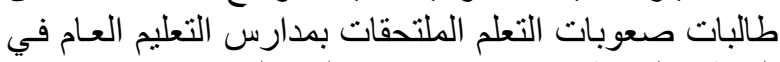
المنطقة الوسطى من 2.3. جمع البيانات 
2.4. بعسض الصــفات الثخصـية للمبحوثــات وعلاقتهـا

بمتوسط كمية اليود المتناول

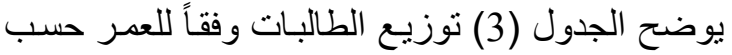

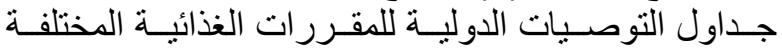
(NRC, 1989). ويبين الجدول أن 82.3\% من الن الطالبـات

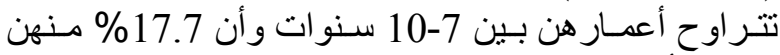

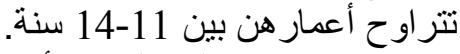

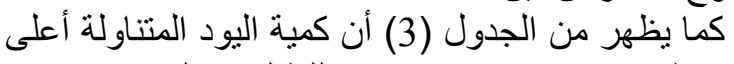

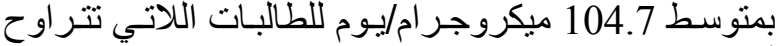

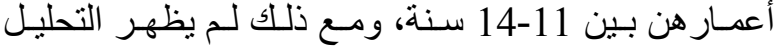

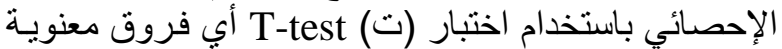

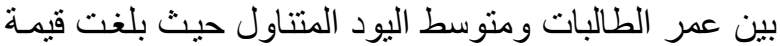

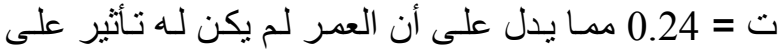

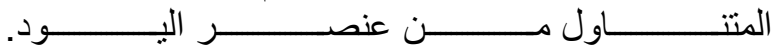

وكان منوسط إفراغ اليود 209.63 ميكروجر ام/جر ام

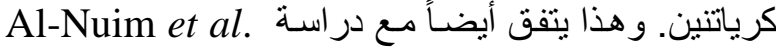

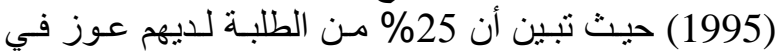

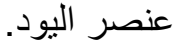

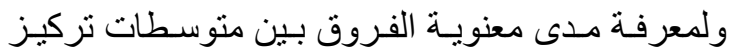
Analysis of اليود في العينات تم استخدام تحليل التبانيل variance

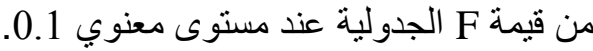

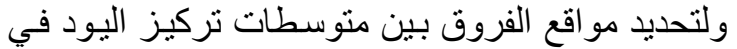

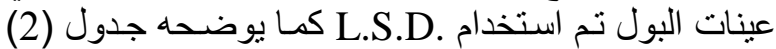

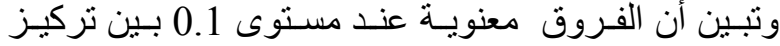

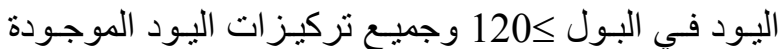

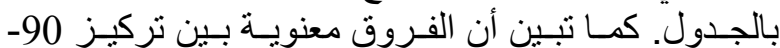

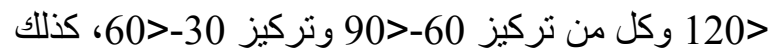

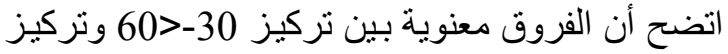

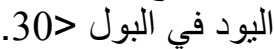

جدول (1): متوسط تركيز اليود (ميكروجرام/جرام كرياتتين) في العينات.

\begin{tabular}{|c|c|c|c|c|}
\hline SD \pm & متوسط تركيز اليود & النسبة المئوية\% & العدد (تكرار) & كرياتتين \\
\hline $9.5 \pm$ & 14.3 & 26.4 & 90 & $30>$ \\
\hline $9.2 \pm$ & 40.7 & 30.0 & 102 & $60>-30$ \\
\hline $8.9 \pm$ & 74.1 & 21.2 & 72 & $90>-60$ \\
\hline $8,8 \pm$ & 105.28 & 4.1 & 14 & $120>-90$ \\
\hline $8.08 \pm$ & 215.29 & 18.3 & 62 & $120 \leq$ \\
\hline & & 100 & 340 & المجموع \\
\hline
\end{tabular}

جدول (2): الفرق بين متوسطات تركيز اليود في العينات وفقاً لتركيز اليود في البول باستخدام (20.S.D.

\begin{tabular}{|c|c|c|c|c|c|}
\hline $30>$ & $60>-30$ & $90>-60$ & $120>-90$ & $120 \leq$ & تركيز اليود في البول \\
\hline 200.99 & 174.59 & ** 141.19 & 110.01 & & $120 \leq$ \\
\hline "90.98 & 64.58 & ${ }^{*} 31.18$ & & & $120>-90$ \\
\hline 59.80 & 33.40 & & & & $90>-60$ \\
\hline 26.40 & & & & & $60>-30$ \\
\hline & & & & & 30> \\
\hline
\end{tabular}

ويعكس الجدول أن متوسط كميـة اليود المتــاول تزيــا

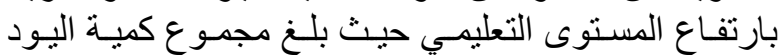

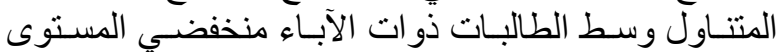

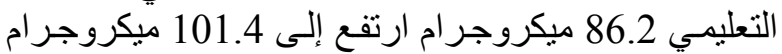

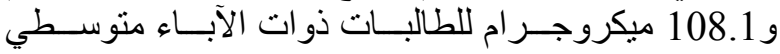
ومرتفعي المستوى التعليمي على التوالي. ويلاحظ انخفاض

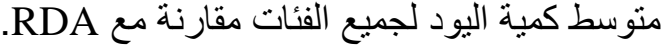
ولمعرفة مدى معنوية الفروق بين متوسطات كمية اليود الفئ

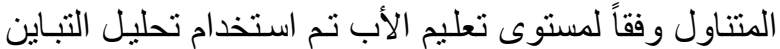
Analysis of variance و هي أعلى من قيمة F الجدولية عند مستوى معنويـة 0.01.

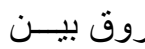

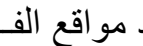
ولتحديـ
جدول (3): متوسط كمية اليود المتناول وفقاً للعمر.

\begin{tabular}{|c|c|c|c|}
\hline 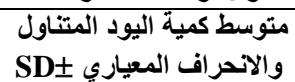 & المئويةًة & (التكرار) & العمر \\
\hline $\begin{array}{c}102.5 \\
(49.1 \pm)\end{array}$ & 82.3 & 280 & 10-7 \\
\hline $\begin{array}{l}104.7 \\
(33 \pm)\end{array}$ & 17.7 & 60 & $14-11$ \\
\hline
\end{tabular}

3.4 3.4 مستوى تعليم الأب

يوضح الجدول رقم (46) مستوى تعليم الأب، حيث تبين

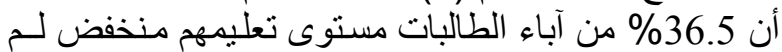

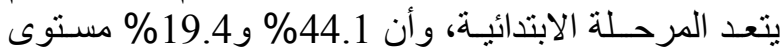
تعليمهم منوسط و عالي على الترتيب. 


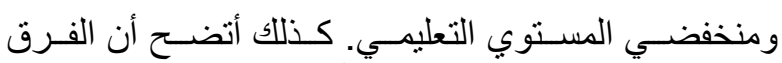

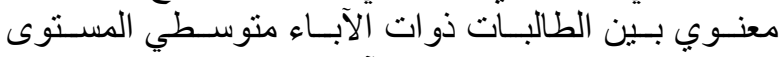

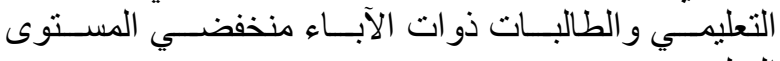

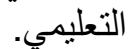

كما L.S.D. متوسطات كمية اليود المتناول تم استخدام

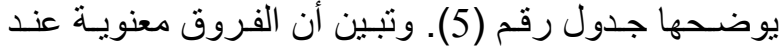

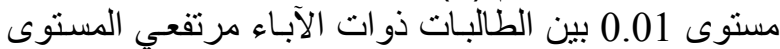

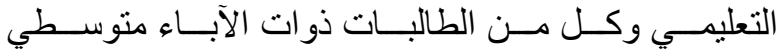

\begin{tabular}{|c|c|c|c|c|}
\hline & & \multicolumn{3}{|c|}{ جدول (4): كمية اليود المتناول وفقاً لمستوى تعليم الأب. } \\
\hline الانحراف المعياري SD士 & $\begin{array}{c}\text { متوسط كمية اليود المتناول) } \\
\text { (ميكروجرام) }\end{array}$ & $\%$ & ع عدد & مستوى تعليم الأب \\
\hline $31.2 \pm$ & 86.2 & 36.5 & 124 & منخفض (ابتدائي وما دون) \\
\hline $40.3 \pm$ & 101.4 & 44.1 & 150 & متوسط (منوسط وثانوي) \\
\hline $47.5 \pm$ & 108.1 & 19.4 & 66 & عالي (جامعي وما فوق) \\
\hline & & 100.0 & 340 & المجموع \\
\hline
\end{tabular}

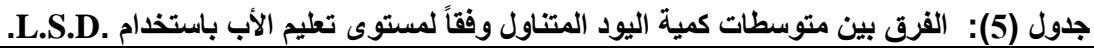

\begin{tabular}{|c|c|c|c|}
\hline منخفض (ابتائي وما دون) & متوسط (متوسط وثانوي) & عالي (جامعي وما فوق) & متوسط تعليم الأب \\
\hline 21.9 & 6.7 & & عالي (جامعي وما فوق) \\
\hline 15.2 & & & متوسط (متوسط وثانوي) \\
\hline & & & منخفض (ابتدائي وما دون) \\
\hline
\end{tabular}

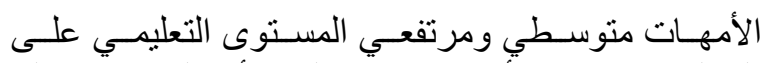

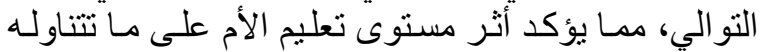

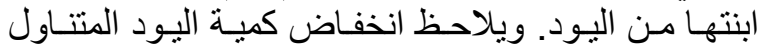

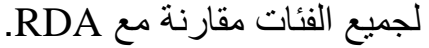

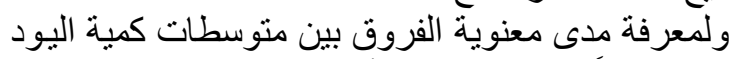

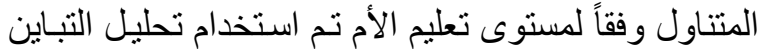

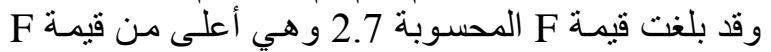
الجدولية عند مستوى معنوية 0.01.
4.4. - مستوى تعليم الأم

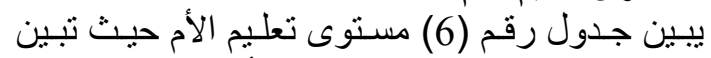

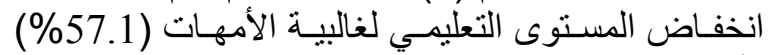

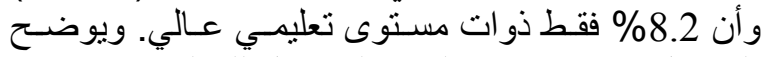

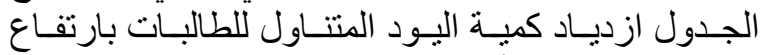

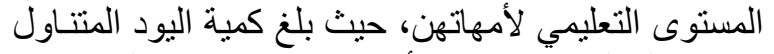

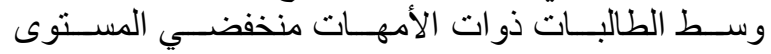

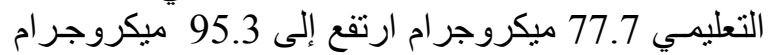
و108.3 ميكروجر ام للطالبات ذوات اتل

جدول (6): كمية اليود المتناول وفقاً لمستوى تعليم الأم.

\begin{tabular}{|c|c|c|c|c|}
\hline SD士 الانحراف المعياري & متوسط كمية اليود المتناول & $\%$ & عدد & مستوى تعليم الأم \\
\hline 34.04 & 77.7 & 57.1 & 194 & منخفض (ابتدائي فما دون) \\
\hline 36.4 & 95.3 & 34.7 & 118 & متوسط (متوسط وثانوي) \\
\hline 45.8 & 108.3 & 8.2 & 28 & عالي (جامعي فما فوق) \\
\hline & & 100.0 & 340 & المجموع \\
\hline
\end{tabular}

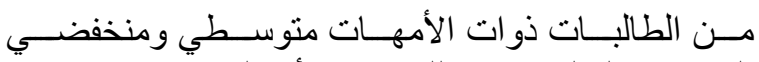

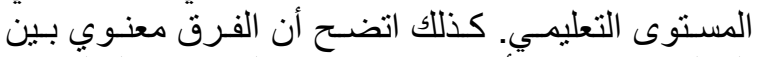

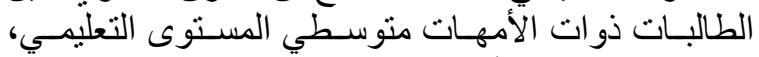

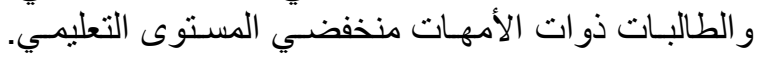

ولتحديـد مو اقع الفروق بـين متوسطات كميـة اليـود

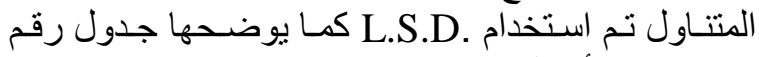

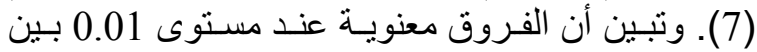
الطالبات ذوات الأمهات مرتفعي المسنتوى التعليمي وكل

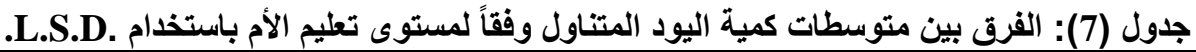

\begin{tabular}{|c|c|c|c|}
\hline منخفض (ابتدائي وما دون) & متوسط (متوسط وثانوي) & عالي (جامعي فما فوق) & متوسط تعليم الأم \\
\hline & & & عالي (جامعي وما فوق) \\
\hline & & 17.6 & متوسط (متوسط وثانوي) \\
\hline & 12.4 & 30.0 & منخفض (ابتدائي وما دون) \\
\hline
\end{tabular}

$$
\begin{aligned}
& \text { 15.9\% مســتوى دخـل أسـرهن هـنـفضض يقـل عـن } \\
& 3000 \text { ربيال شهرياً. } \\
& \text { ويعكس الجدول أن متوسط كمية اليـود المتناول }
\end{aligned}
$$

$$
\begin{aligned}
& \text { يوضح جدول (8) مستوى دخل أسر الطالبات حيث }
\end{aligned}
$$

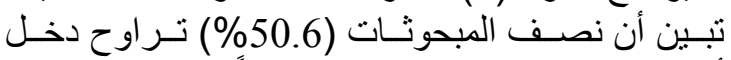

$$
\begin{aligned}
& \text { أسر هن من 3000-6000 بيال شهرياً مقابل }
\end{aligned}
$$


قيمة F الجدولية. ولتحديد مو اقع الفروق بين منوسطات كمية

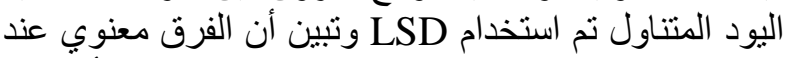

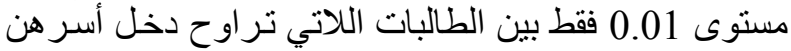
بين 3000-6000 ربال وكـل مـن الطالبـات اللاتـي تـر اوح دخل أسـرهن أقل مـن 3000 ريال وكل و والتي تزيد عن 6000

نتيجة لانخفاض مستوى اليود لطالبات صعوبات التعلم

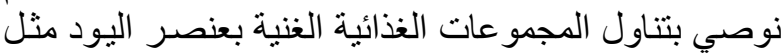
الأسمآك وبعض الأغذية الأخري.

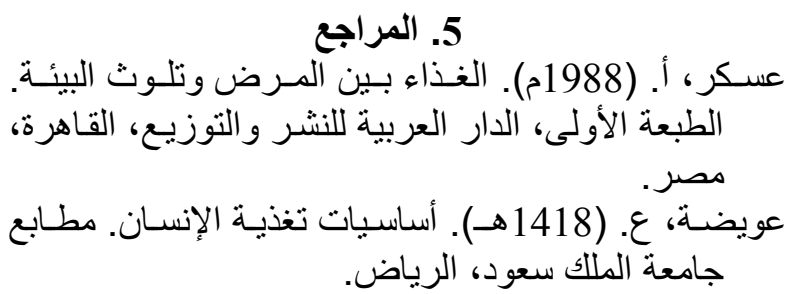

AI-Attas O. and Sulimani R. (1993) Iodine concentrations in Saudi staple foods. Saudi Med. L, 14: 322-324.

Al-Nuim A., Al-Mazrou Y., Farag M. and AlAttas O. (1995). Deficiency Disorders Survey Saudi Arabia. Kingdom of National Iodine Saudi Arabia Ministry of Health, Riyadh.

Brasward J., Hulshof K. and Lowik M. (1995). calculated iodine intake before and after simulated iodization (Dutch Nutrition Surveillance System). Ann. Nutr. Metab., 39:58-94.

Dunn J. and Haar F. (1990). A practical guide to the correction of iodine deficiency, International Council for Control of Iodine Deficiency Disorders. (1990).

Dunn J., Crutchfield H., Gutekunst R. and Dunn A. (1993). Two simple methods for measuring iodine in urine. Thyroid., 3:119123.

Delange F., Bruno B., Hans B. (2002). Determining median urinary iodine concentration that indicates adequate iodine intake at population level. Bull. World Health Org., 80(8): 633-636.

FNB NRC (Food and Nutrition Board, National Research Council). (1989). Recommended Dietary Allowances. $10^{\text {th }}$ ed. Washington, D.C. National Academy of Sciences.

Gibson R.S. (1990). Principles of Nutrition Assessment. Oxford University Press, New York.

\begin{tabular}{|c|c|c|c|c|}
\hline \multicolumn{5}{|c|}{ 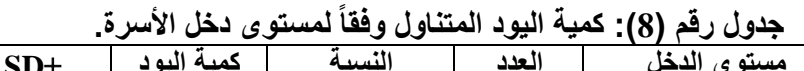 } \\
\hline SD \pm & كية اليود & 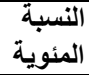 & (تلعرار) & $\begin{aligned} \text { (ريال) } \\
\text { (رستوى الاخل }\end{aligned}$ \\
\hline 36.2 & 94 & 15.9 & 54 & 3000> \\
\hline 46.9 & 10 & 50.6 & 172 & $6000-3000$ \\
\hline 38.08 & 94.2 & 33.5 & 114 & $6000<$ \\
\hline & & 100.0 & 340 & וمج" \\
\hline
\end{tabular}

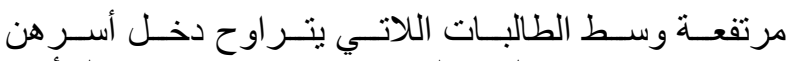
بين3000-6000 ريال إذ بلغ 107.3 ميكروجر ام مقابل دابل أكثر

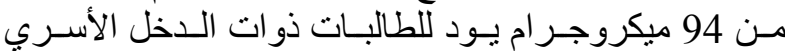

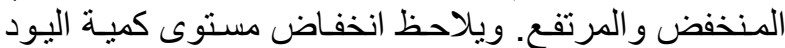

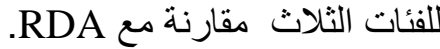

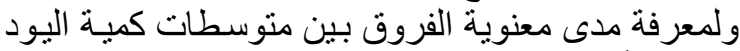
المتناول وفقاً لمستوى دخل الأسرة تم استخدام تحليل التباين، وقد بلغت قيمة F المحسوبة 3.30 و هي أعلى من الن

Gushurst C., Mueller J. and Sedor F. (1984). Breast milk iodine: Reassessment in 1980s. Pediatrics, 73:354-357.

Guthrie, H. (1983). Introductory Nutrition, The C.V. Mosby Company, United States of America.

Hollowed J., Staehing N., Hannon W., Flander D., Gunter E., Maberly G., Braverman L., Pino S., Miller D., Garbe P., Delozier D. and Jackson R. (1989). Iodine Nutrition in the United States. J. Clin. Endocr. And Metab., 83(10):34013408.

Hunt S. and Groff J. (1990). Advanced Nutrition and Human Metabolism. West Publishing Company, New York. Jolin T. and Escobar del Ray F. (1965). Evaluation of iodine / creatinine ratios of casual samples as indices of daily urinary iodine out put during field studies. J. Clin. Endocrinol. Metab. Vol. 25:540542.

Jolin T. and Escobar del Ray, F. (1956). Evaluation of iodine / creatinine ratios of casual samples as indices of daily urinary iodine out put during field studies. J. Clin. Endocrinol. Metab.. (25):540-542.

Murray R., Daryl K., Peter A. and Victor W. (1991). Harper's Biochemistry, Libraties du.

Nieman D., Butterworth D. and Nieman C. (2001). Nutrition. First Edition, Wm. C. Brown Publishers. USA. 
NRC (National Research Council). (1989). National Academy of Sciences Recommended Dietary Allowances. 10 $0^{\text {th }}$ ed. National Academy of Sciences. Washington, D.C.

Pierre V., Zarebska M., Prezoisi P., Galan P., Pelletier B. and Hercberg S. (1999). Iodine deficiency in France. Lancet., 353:1766-1769.

SAS (1995). SAS User's Guide: Statistics, SAS Institute, Inc., Cary, NC.

Sandell E.B. and Kolthoff I.M. (1937) Micro determination of iodine by a catalytic method. Micro Chime Acta, 1: 9-25.

Strove C. and Ohlen S. (1990). Einflub Fruherer Schwangerchften auf strumaund khotenhau figkeit bei Schilddrusengensunden frauen. Deutsch. E. Medizinische Wochen Scrift., 115:1050-1053. C.F.

Stubbe P., Al-Habib S. and Thal H. (1988). Iodine excretion and goiter prevalence in Saudi Arabian children. Saudi Med. J., 9(5):476-480.

Sulimani R., Al-Attas O., Desouki M., Al-

Nuaim A. and Al-Sekait May (1991). Iodine concentrations in Saudi water. Annuals of Saudi Med., 6:655-656.
Sayed N., Sally M., Khan M. and Andrew T. (1999). Biochemical hypothyroidism secondary to iodine deficiency is associated with poor school achievement and cognition in Bangladeshi children. J. Nutr. 128:980987.

Underwood E.J. (1977). Trace elements in human and animal nutrition. Academic Press, New York. USA.

Vought R., London W. and Stebbing G. (1967). Endemic goiter in North Virginia, J. Clin. Endocr. Metab., 27:1381-1389.

WHO (World Health Organization). (1988). Guidelines for a national programme for the control of iodine deficiency disorders in eastern Mediterranean region. Regional Office for the Eastern Mediterranean.

WHO (World Health Organization). (1991). Evaluation of eastern food additives and contaminants. Thirtyseventh report of a joint FAO/WHO Expert Committee on Food Additives. Geneva.

Wenlock R., Buss D., Moxon R. and Bunton N. (1982). Trace nutrients iodine in British food. Br. J. Nutr. 47:381-390. Nutr. 47:381-390. 